\title{
RESEARCH
}

Open Access

\section{Effect of platelet-rich and platelet-poor plasma on 3D bone-to-implant contact: a preclinical micro-CT study}

Dandan Song ${ }^{1,2^{*}}$ (D), Sohaib Shujaat ${ }^{1,2}$, Yan Huang ${ }^{1,2,3}$, Jeroen Van Dessel ${ }^{1,2}$, Constantinus Politis ${ }^{1,2}$, Ivo Lambrichts ${ }^{4}$ and Reinhilde Jacobs ${ }^{1,2,5}$

\begin{abstract}
Background: Bone-to-implant contact ratio (BIC\%) plays a critical role in secondary stability of osseointegrated dental implants. The aim of this study was to identify the correlation of 2D/3D micro-CT images with histology as a gold standard for evaluating $\mathrm{BIC} \%$ and to investigate the influence of the platelet-rich plasma (PRP) and platelet-poor plasma (PPP) on 3D BIC\% following delayed implant placement with delayed loading (DIP+DL).

Methods: Nine beagle dogs were recruited. Following bilateral extraction of mandibular 3rd premolar, 4th premolar, and 1st molar, 54 screw-type titanium implants were inserted and randomly divided into one control and two test groups based on a split-mouth design. The control group involved DIP+DL $(n=18)$ and both test groups included DIP+DL with local application of PRP $(n=18)$ and PPP $(n=18)$. A BIC analysis was performed utilizing 2D histomorphometry and 2D/3D micro-CT. Following identification of correlation between histology and 2D/3D micro-CT images, a 3D micro-CT assessment of the 3D BIC\% at three follow-up timepoints (1,3, and 6 months) was carried out for observing the influence of PRP and PPP on BIC.

Results: The 2D micro-CT BIC\% values revealed a strong positive correlation with histology $(r=0.98, p<0.001)$ and a moderate correlation existed with 3D micro-CT $(r=0.67, p=0.005)$. BIC levels at 1 month and combined influence of PPP and PRP irrespective of time-points revealed significantly higher 3D BIC\% compared to the control. However, a reduction in 3D BIC\% was observed at the 3rd and 6th month. No significant difference was observed between both PRP and PPP.

Conclusions: Both $2 \mathrm{D}$ and $3 \mathrm{D}$ micro- $\mathrm{CT}$ demonstrated a potential to be utilized as a complimentary method for assessing BIC compared to the histological gold standard. Overall, both PRP and PPP significantly facilitated bone healing and osseointegration with a higher 3D BIC at follow-up. However, their influence was reduced as the observation period was increased.
\end{abstract}

Keywords: Platelet-rich plasma, Osseointegration, Bone-implant interface, X-ray micro-CT, Dental implants

\footnotetext{
* Correspondence: dandansong9015@gmail.com

'OMFS IMPATH Research Group, Department of Imaging \& Pathology,

Faculty of Medicine, KU Leuven, Kapucijnenvoer 33, 3000 Leuven, Belgium

${ }^{2}$ Oral and Maxillofacial Surgery, University Hospitals Leuven, Leuven, Belgium

Full list of author information is available at the end of the article
}

\section{Springer Open}

(0) The Author(s). 2021 Open Access This article is licensed under a Creative Commons Attribution 4.0 International License, which permits use, sharing, adaptation, distribution and reproduction in any medium or format, as long as you give appropriate credit to the original author(s) and the source, provide a link to the Creative Commons licence, and indicate if changes were made. The images or other third party material in this article are included in the article's Creative Commons licence, unless indicated otherwise in a credit line to the material. If material is not included in the article's Creative Commons licence and your intended use is not permitted by statutory regulation or exceeds the permitted use, you will need to obtain permission directly from the copyright holder. To view a copy of this licence, visit http://creativecommons.org/licenses/by/4.0/. 


\section{Background}

Implant osseointegration has been described as the simultaneous contact and distant osteogenesis at the implant surface and osteotomy wall leading to new bone synthesis and a direct bone-to-implant contact (BIC) without intervening connective tissue [1]. The amount of BIC is an important determinant for achieving optimal secondary implant stability [2]. Amongst different methods proposed in literature for evaluating BIC, the $\mathrm{BIC}$ ratio $(\mathrm{BIC} \%)$ is one of the most commonly utilized objective methods for quantifying the extent of osseointegration following implant placement [3]. It measures the bone in-growth in the transitional region and is defined as the percentage of the bone in contact with the implant surface. The success of implant is ensured when the $\mathrm{BIC} \%$ is maintained at a minimum of $50-65 \%[4,5]$. Thereby, the fundamental goal of implant therapy is to obtain a favorable BIC to prevent implant micromobility and for maximizing the implant survival rate.

The two most commonly utilized imaging modalities for evaluating $\mathrm{BIC} \%$ in animal models include histomorphometry and micro-computed tomography (micro-CT) $[6,7]$. Although histomorphometry is a reliable method and has been utilized as a gold standard for measuring 2D BIC\%, information acquired through twodimensional (2D) slices is deemed insufficient for representing the three-dimensional (3D) bone structure [8]. To overcome the limitations associated with histomorphometry, micro-CT has been recommended for assessing $\mathrm{BIC} \%$ based on its rapidity, reproducibility, and non-destructiveness [9]. Most of the evidence focusing on micro-CT assessment of $\mathrm{BIC} \%$ are pseudo threedimensional (3D) in nature, as instead of analyzing the true reconstructed $3 \mathrm{D}$ image, the measurements are performed on 2D cross-sectional images [10, 11]. Although, cross-sectional images are pseudo 3D, still they form a basis for validating the accuracy of micro-CT crosssectional measurements compared to a histological reference.

In literature, various parameters have been reported to influence the BIC levels, such as, implant type and size $[12,13]$, implant surface treatment [14], bone quality and quantity [15], implant loading conditions [16, 17], and forces exerted on implant [18, 19]. Attempts have been made for enhancing the BIC values by modifying the roughness, composition, and surface treatment of the dental implants. Recently, multiple growth factors, most commonly, platelet-derived growth factors have been utilized for enhancing the BIC [20]. Some studies have shown improved implant stability and earlier osteogenesis with plasma containing higher concentration of platelets $[21,22]$. However, there is lack of evidence related to the influence of different platelet-derived plasma concentrations on the 3D BIC at follow-up.
Therefore, the following animal study was conducted to address two aims. The first aim was to identify the correlation of 2D/3D micro-CT images with histology as a gold standard for evaluating $\mathrm{BIC} \%$. The second aim investigated the influence of the platelet-rich plasma (PRP) and platelet-poor plasma (PPP) on 3D $\mathrm{BIC} \%$ following delayed implant placement with delayed loading (DIP+ DL) utilizing micro-CT.

\section{Methods}

\section{Animal model and sample size}

The protocol for this study was approved by the Bioethics Committee of Sichuan University (reference No. WCCSIRB-D-2014-010) and complied with the ARRIVE guideline for preclinical studies [23]. Nine beagle dogs (weight 14-17 kg, age 12-14 months) were recruited. An identical housing and feeding condition were executed for all the animals at the Experimental Animal Center of Laboratory of Biotherapy. The sample size was in accordance with the previous studies [24-26] and also based on a priori power analysis in $\mathrm{G}^{*}$ power 3.1 at a power of $80 \%$ and 0.05 level of significance [27].

\section{PRP and PPP preparation}

A volume of $5 \mathrm{ml}$ venous blood was collected from the cephalic vein of one of the forelegs of each experimental dog. The samples were transferred into sterile tube containing $1 \mathrm{ml}$ of sodium citrate which acted as an anticoagulant and placed into a centrifuge (Allegra X 30R centrifuge, CA, USA). A previously optimized doublecentrifugation protocol was applied for the preparation of PRP and PPP [28]. The first centrifugation involved a relative centrifugal force (RCF) of $700 \mathrm{~g}$ for $8 \mathrm{~min}$ and a second one at a RCF of $1600 \mathrm{~g}$ for $8 \mathrm{~min}$. Following separation, $1 \mathrm{ml}$ of each PRP and PPP were extracted from the sample. Both PRP and PPP were stored at room temperature in a conventional shaker until use.

\section{Surgical procedure}

Each dog was administered with a 1-week prophylactic antibiotic therapy (gentamycin sulphate $300 \mathrm{mg}$ ) both before and after surgery for preventing infection. All surgeries were performed under general $(0.1 \mathrm{ml} / \mathrm{kg}$ xylazine hydrochloride) and local anesthesia (2-4 ml lidocaine $2 \%$ epinephrine). A bilateral extraction of mandibular 3rd premolar, 4th premolar, and 1st molar was performed. After 1-month healing time, 54 screw-type titanium dental implant with plasma-sprayed hydroxyapatite (HA) coating (3.3 mm $\varnothing \times 8 \mathrm{~mm}$, cylindrical, non-submerged healing, BLB, China) were inserted at the healed extraction sites ( $n=6$ per dog). The implants were randomly divided into one control and two test groups $(n=18$ per group) based on a split-mouth design. The control group involved DIP+DL $(n=18)$ and the two test groups 
included DIP+DL with local application of PRP $(n=18)$ and PPP $(n=18)$. Each implant in the test groups was dipped in PPP or PRP solution prior to insertion in the alveolus. Implants were inserted with a controlled insertion torque ranging between 30 and $35 \mathrm{~N} \mathrm{~cm}$. All surgical procedures were performed by an experienced oral and maxillofacial surgeon who was blinded to the allocation process. A resin crown was fabricated and placed onto each implant following 1 month of treatment with a resin cement (RelyX, Unicem, RX, 3 M ESPE, St. Paul, USA) under halogen light-curing unit for $20 \mathrm{~s}$. Afterwards, an articulating paper (20- $\mu \mathrm{m}$ thick, Accufilm II, RX, 3M ESPE, St. Paul, USA) was used to check the contacts and make occlusal adjustments of the prosthetic crown to prevent possible overloading with the opposing natural teeth.

\section{Three-dimensional micro-CT assessment}

A high-resolution micro-CT (Quantum FX Caliper, Life Sciences, Perkin Elmer) was utilized for acquiring radiographic images of the mandibular bone blocks with implants from each animal and analyzing $\mathrm{BIC} \%$ follow-up changes at 1- (T1), 3- (T3), and 6-month (T6) timepoints. The acquisition protocol included $20-\mu \mathrm{m}$ pixel size, $360^{\circ}$ rotation, $90-\mathrm{kVp}$ tube voltage, $160-\mu \mathrm{A}$ tube current, 180 -s scanning time, and a $20-\mathrm{mm}^{2}$ field of view (FOV). All the images were manually reoriented along the long axis of the implant in axial, coronal, and sagittal plane utilizing Dataviewer software (Ver. 1.5.1.2; Bruker-
CT; Kontich, Belgium). Thereafter, image processing was performed with CT Analyser software (version 1.16.1.0, Skyscan1272, Bruker Microct, Kontich, Belgium). The whole implant body was included in the volume of interest (VOI).

The implant shoulder was set as the reference for the superior limit of the VOI and the apex of the implant as the inferior limit (Fig. 1). The height of all the VOIs were kept constant for standardizing its vertical limit. Following determination of a circular region of interest (ROI) and binarization, a segmentation algorithm with separate histogram thresholding was applied for creating a distinction between implant (minimum, 43; maximum, 100 ) and bone (minimum, 110; maximum, 255) and calculating 3D BIC [29]. The BIC at a distance of 5 pixels from the implant surface was selected and $\mathrm{BIC} \%$ was automatically calculated and represented by the percent intersection surface (I.S/TS) (Fig. 1).

\section{Histological assessment}

Based on a random direct sampling technique, the dogs from each group were randomly euthanized at T1, T3, and T6 with an overdose of an intravenous injection of xylazine hydrochloride. Thereafter, bone blocks were prepared and decalcified with ethylenediaminetetraacetic acid (EDTA, $0.5 \mathrm{~mol} / \mathrm{L}$ ) and phosphate-buffered saline (pH 7.4) at $4{ }^{\circ} \mathrm{C}$ for 10 months. Care was taken to avoid damage to the samples while removing implants with surgical forceps. The bone blocks were dehydrated and

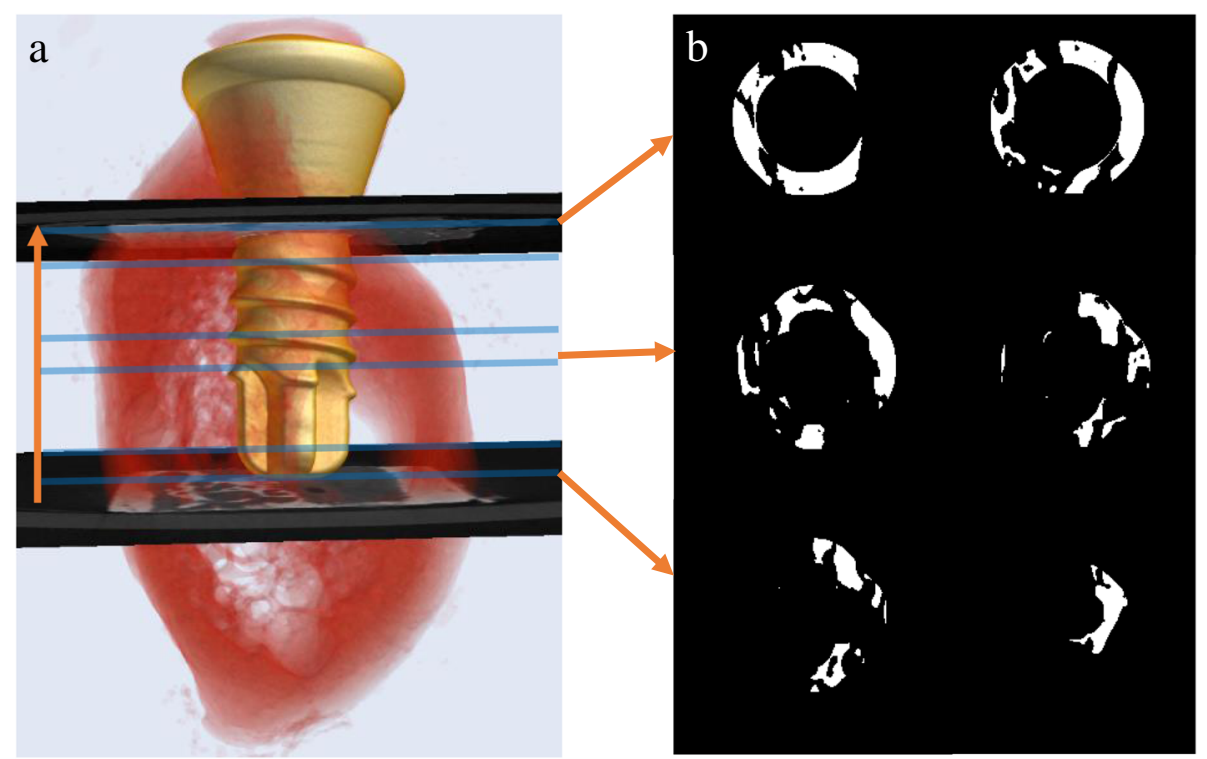

Fig. 1 Automatic micro-CT-based evaluation of bone-to-implant contact (BIC) around the implant. a 3D surface reconstruction indicating the superior (implant shoulder) and inferior (implant apex) limit of volume of interest. $\mathbf{b}$ Binary images of BIC surface based on a custom processing with CTAn (version 1.16.1.0, Skyscan1272, Bruker Microct, Kontich, Belgium). Orange arrows indicate the series of slices from the implant apex to the shoulder level. Blue lines indicate the position of the images in $\mathbf{b}$ which show the amount of peri-implant bone descends from apex to coronal region 
fully infiltrated with paraffin and sliced into thin serial sections $(\sim 6 \mu \mathrm{m})$ in a bucco-lingual direction. All slices were stained with Masson's trichrome stain. The BIC\% was calculated based on a previously validated protocol [29].

Following selection of the matching histology slices and $2 \mathrm{D}$ micro-CT images, they were spatially aligned and registered for evaluating the same region as suggested by Soares et al. [30]. The BIC\% was calculated by assessing the entire implant surface and contact areas between bone and implant using AxioVision software (Version 4.7.1; Carl Zeiss MicroImaging GmbH, Jena, Germany) (Fig. 2).

\section{Statistical analysis}

The data analysis was performed using the SPSS software (Version 22, IBM, NY, USA). Normality of data distribution was assessed with the Shapiro-Wilk test. All the data showed normal distribution, thereby, parametric tests were utilized for assessing significance. The correlation between the 2D/3D micro-CT and histological $\mathrm{BIC} \%$ was performed with the Pearson correlation coefficient. Afterwards, mean and standard deviation of the 3D micro-CT $\mathrm{BIC} \%$ was calculated. Analysis of variance (ANOVA) and post hoc Bonferroni tests were employed for calculating the significance of difference between groups, different times points, and combined effect independent of time-points $(\alpha=0.05)$.

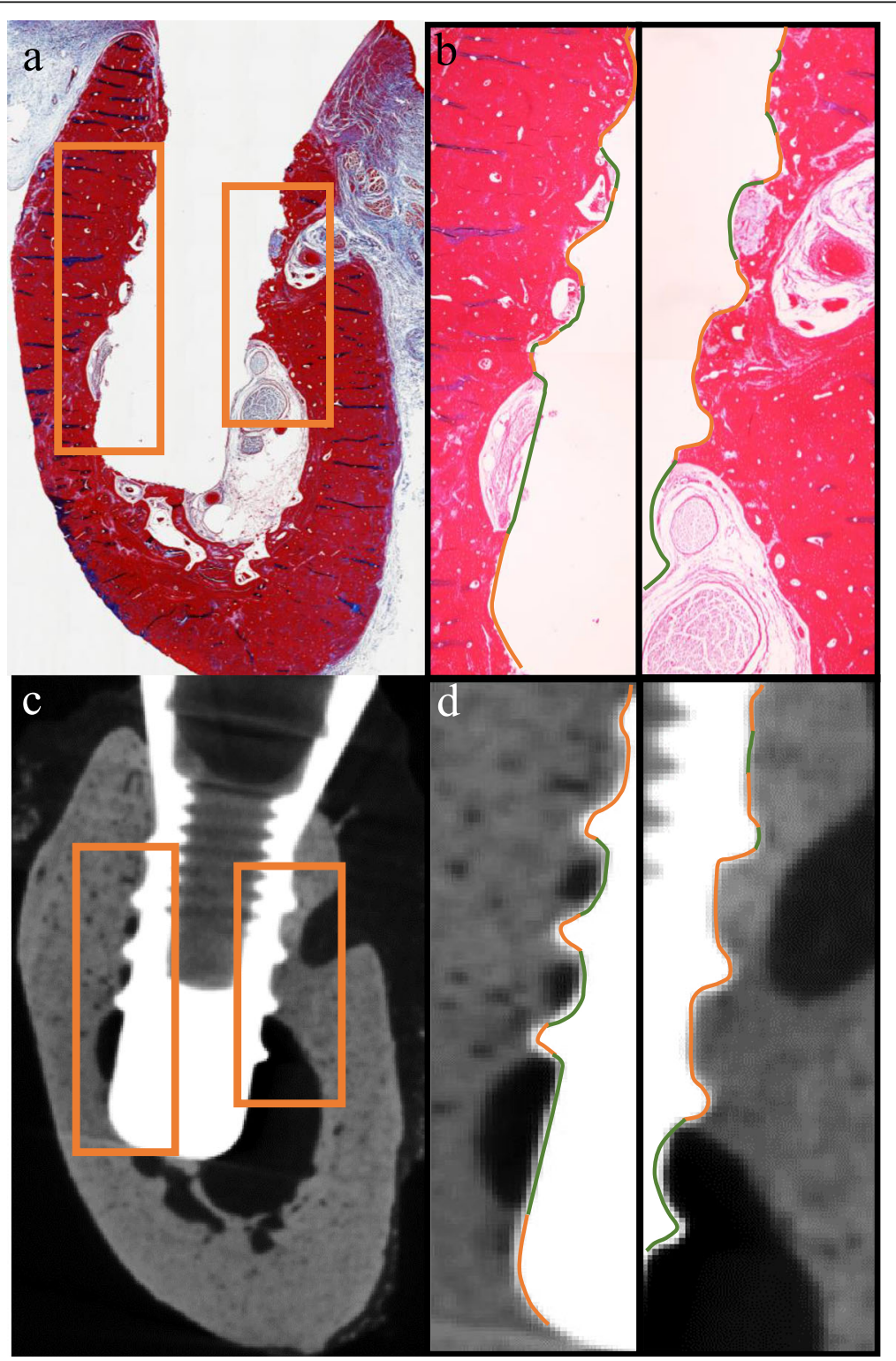

Fig. 2 Matching sections of histology and micro-CT image and BIC\% measurement. a, c Matching bucco-lingual images of histology and microCT. $\mathbf{b}$, d Magnification of the orange rectangle in $\mathbf{a}$ and $\mathbf{c}(\times 40)$. Orange line is the bone-to-implant contact; green line is the implant thread 


\section{Results}

All dogs remained in good health during the experiment. The implants in all groups were clinically stable till euthanasia with no sign of infection or any complication. The $2 \mathrm{D} \mathrm{BIC} \%$ values obtained from matching micro-CT and histology images revealed a strong positive significant correlation, regardless of the group or time-point $(r=0.98, p<0.001)$. However, a moderate correlation existed between 3D micro-CT and histology images $(r=$ $0.67, p=0.005$ ).

Table 1 describes the 3D $\mathrm{BIC} \%$ of the control and test groups at the 1st, 3rd and 6th month observation timepoints. At the 1st month of healing time, PPP $(p=0.09)$ and PRP ( $p=0.039)$ test groups showed the highest 3D $\mathrm{BIC} \%$ with a significant increase compared to the control group. However, no significant increase was observed at the 3rd and 6th month. Both test groups showed a decline in $3 \mathrm{D} \mathrm{BIC \%}$ at the 3rd and 6th month compared to the 1st month, and the 6th month time-point showed a further decrease in 3D BIC\% compared to the 3rd month without any significant difference. At the 6th month time-point, the PPP test group showed higher 3D BIC\% $(55.3 \pm 8.7 \%)$ compared to PRP $(52.9 \pm 7.5 \%)$ and the control group $(51.5 \pm 4.7 \%)$ without any significant difference.

Overall, the $3 \mathrm{D}$ BIC\% was found to be significantly high in PRP $(63.8 \pm 4.4 \%, p=0.024)$ compared to the control $(51.5 \pm 4.7 \%)$ irrespective of the time-point (Fig. 3), with PRP group showing the most increase in 3D BIC\%. No significant difference was observed between PPP and PRP group.

\section{Discussion}

In our study, a strong correlation existed between 2D micro-CT and histology images, whereas with the utilization of 3D micro-CT, a moderate correlation was observed with a significant difference in $\mathrm{BIC} \%$ of up to $4.9 \%$. This minimal bias could be attributed to the absence of 3D BIC information in histology which varies depending on the implant surface being evaluated. Additionally, as the histological slices are taken at certain intervals at pre-selected locations which have a risk of misrepresenting, the entire osseous situation. Thereby, both $2 \mathrm{D}$ micro-CT and histological slices are unable to represent the true 3D bone structure around the implant. Our findings were consistent with prior studies which also indicated a strong correlation between 2D micro-CT and histology and a moderate correlation between 3D micro-CT and histology [11, 31]. For overcoming the limitations of $2 \mathrm{D}$ histological and pseudo 3D micro-CT images, we utilized a highresolution 3D micro-CT-based methodology for calculating the $3 \mathrm{D} \mathrm{BIC} \%$ by excluding four pixels closest to the implant. These steps ensured standardized $\mathrm{BIC} \%$ assessment without under- and over-estimation and overcame the influence of partial volume effect around the trabecular edges and beam hardening near the implant surface.

The 3D BIC\% was assessed at different follow-up time-points following application of PRP and PPP compared to the control group. Our findings suggested a significant increase in $\mathrm{BIC} \%$ at the 1st month following both PRP and PPP application, thereafter a slight decrease was observed at follow-up. Studies have shown significantly higher $\mathrm{BIC} \%$ at an early healing phase following PRP application, which was in accordance with our findings [32]. This might be explained based on the short life-span of platelets (approximately 5-10 days) [33]. A high concentration of platelet growth factors is initially secreted within the first $10 \mathrm{~min}$ following blood clotting and within the first hour, over $95 \%$ of the presynthesized growth factors contained in the alphagranules complete their secretion [34]. Following initial burst release of PRP-derived growth factors, they continue to synthesize and secrete additional growth factors during their remaining life-span $[35,36]$. These growth factors promote and accelerate tissue healing and regeneration [37, 38]. Additionally, the centrifugated platelets in the form of PRP also accelerate cell proliferation and bone healing. However, no studies were found assessing the influence of PPP which requires further investigations. The long-term influence of plasma leading to significant enhancement of bone formation in intra-bony periodontal defects and sinus augmentation has been well-documented [39-41]. However, it remains unclear and controversial whether long-term effect of PRP and PPP can be regarded as clinically favorable or not in implant therapy.

Table $13 \mathrm{D}$ bone-implant contact ratio of control (DIP+DL) and test groups (DIP+DL+PPP, and DIP+DL+PRP) at different follow-up time-points using micro- $C T\left({ }^{*} p<0.05\right)$

\begin{tabular}{|c|c|c|c|}
\hline Observation period & Group & Bone-implant contact, mean \pm SD (\%) & $\begin{array}{l}p \text { value; (1) vs (2), (1) vs (3), } \\
\text { (2)vs (3) }\end{array}$ \\
\hline 1 month & (1) $\mathrm{DIP}+\mathrm{DL}$, (2) DIP+DL+PPP, (3) DIP+DL+PRP & $52.2 \pm 3.2,69.5 \pm 2.6^{*}, 73.4 \pm 5.6^{*}$ & $0.09 *, 0.039 *, 1$ \\
\hline 3 months & (1) $\mathrm{DIP}+\mathrm{DL}$, (2) DIP+DL+PPP, (3) DIP+DL+PRP & $47.3 \pm 11.2,59.0 \pm 2.7,65.2 \pm 4.0$ & $1,0.513,0.819$ \\
\hline 6 months & (1) $\mathrm{DIP}+\mathrm{DL}$, (2) DIP+DL+PPP, (3) DIP+DL+PRP & $54.9 \pm 7.7,55.3 \pm 9.1,52.9 \pm 7.1$ & $1,1,1$ \\
\hline Overall & (1) $\mathrm{DIP}+\mathrm{DL}$, (2) $\mathrm{DIP}+\mathrm{DL}+\mathrm{PPP}$, (3) $\mathrm{DIP}+\mathrm{DL}+\mathrm{PRP}$ & $51.5 \pm 4.7,61.3 \pm 4.8, \mathbf{6 3 . 8} \pm \mathbf{4 . 4 ^ { * }}$ & $0.078, \mathbf{0 . 0 2 4 *}, 1$ \\
\hline
\end{tabular}




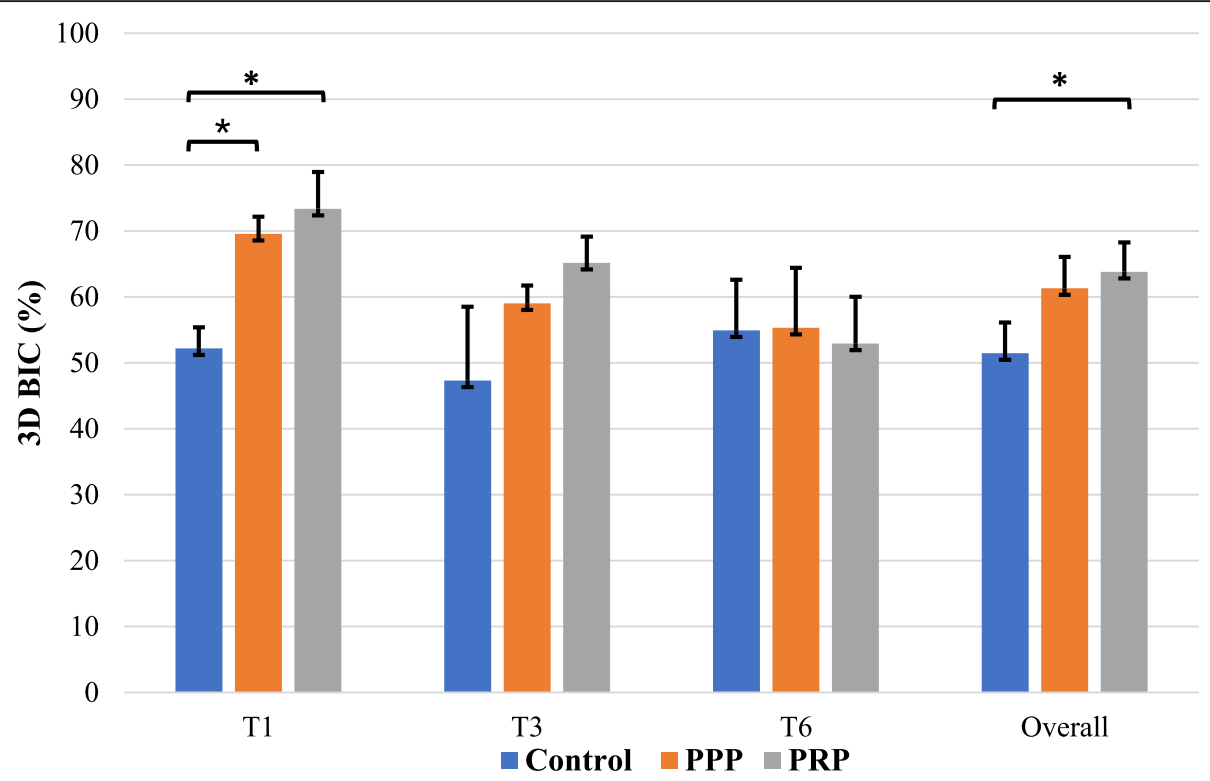

Fig. 3 Impact of PRP and PPP on 3-demension bone-implant-contact ratio (3D BIC\%) at individual time-points and overall influence irrespective of time. PPP, platelet-poor plasma; PRP, platelet-rich plasma; T1, 1 month; T3, 3 months; T6, 6 months. ${ }^{*} p<0.05$ statistically significant

When considering the overall combined impact of PPP and PRP irrespective of the time interval, an obvious increase in $\mathrm{BIC} \%$ was observed with more than $60 \%$ in both test groups. Our findings were in an agreement with some studies [32, 42, 43]; at the same instance, inconsistencies were observed with other studies suggesting no influence of PPP or PRP on new bone formation [44-46]. The possible explanation for the inconsistent findings could be due to the different centrifugation techniques which can lead to substantial difference in platelet, leucocyte, and growth factors level. This requires further research to find an optimal standardized method for preparing plasma and later on studying which factors can lead to higher implant stability. The concentration of platelet in PRP $\left(776.2 \pm 144.0 \times 10^{9} / \mathrm{L}\right)$ is almost 2000 times higher than that in PPP $(4.8 \pm 1.8$ $\times 10^{9} / \mathrm{L}$ ) [22]. However, in our study, both PRP and PPP have positive effect on promoting bone regeneration independent of the amount of platelet concentration. As the number of fibrin fibers is greater in PPP compared to PRP [47], which could explain the final biological bone healing effect of PPP during which lower platelet count is compensated by the fibrin fibers. The potential of these fibers acting to provide a scaffold for supporting cells and releasing growth factors should not be ignored. In some studies, PPP was discarded based on its low platelet nature $[48,49]$. We believe, based on its potential in speeding up the bone regeneration regardless of the platelet concentration, future studies should also focus on the long-term impact of PPP on BIC.

The study had certain limitations. Firstly, primary implant stability was not evaluated. Secondly, the bone blocks underwent decalcification prior to histological preparation which might have impacted the BIC calculation, yet researchers carefully removed the implant after decalcification ensuring no further harm to the periimplant bony surface. Thirdly, the small sample size could have led to bias within our findings. Future preclinical studies with a larger sample size should focus on quantifying the combined impact of different plasma preparation techniques, implant treatment protocols, bone density, and other modified implant surfaces on long-term 3D BIC\%. Additionally, further animal research should be carried out assessing the influence of PRP and PPP following implant placement in compromised bone defects. Later on, the results of this analysis could then be applied and tested in patients with poor wound healing.

\section{Conclusions}

Both 2D and 3D micro-CT demonstrated a potential to be utilized as a complimentary method for assessing BIC compared to the histological gold standard. However, further studies should be considered for improving the correlation of $3 \mathrm{D}$ micro-CT with histological images. Overall, both PRP and PPP were able to contribute towards a significantly higher BIC following implant treatment. However, their influence was reduced as the observation period was increased, with BIC having the highest ratio at the 1st month and lowest at 6 months. It can be assumed that the plasma-fibrin may facilitate and speed up bone healing and osseointegration. 


\section{Abbreviations}

BIC\%: Bone-to-implant contact ratio; 2D: Two-dimensional; 3D: Threedimensional; PRP: Platelet-rich plasma; PPP: Platelet-poor plasma; DIP+DL: Delayed implant placement with delayed loading; micro-CT: Microcomputed tomography; RCF: Relative centrifugal force; FOV: Field of view; VOI: Volume of interest; ROI: Region of interest; HA: Hydroxyapatite

\section{Acknowledgements}

The authors are thankful to the laboratory technicians from the Research Base of West China Hospital, Sichuan University, and Translational Cell and Tissue Research of University Hospitals Leuven, KU Leuven, for their valuable help during this animal research.

\section{Authors' contributions}

All authors have read and approved the final manuscript. HY, CP, and RJ designed this study. HY did the animal experiment. IL helped with the histological staining. SD performed the measurements. JVD did the statistical analysis. SD and SS wrote the first draft of the manuscript. SS and RJ corrected the manuscript.

\section{Funding}

This work was supported by a Sichuan Province Science and Technology Support Program (2016SZ0010). DS received fellowship support from China Scholarship Council (201708210187). HY is a postdoctoral (11.N3.615N) and JVD is a predoctoral (11.ZU.117N) FWO-research fellow.

\section{Availability of data and materials}

The datasets used and/or analyzed during the current study are available from the corresponding author on reasonable request.

\section{Ethics approval and consent to participate}

This animal experiment was approved by the Bioethics Committee of Sichuan University (reference number WCCSIRB-D-2014-010).

\section{Consent for publication}

$$
\text { Not applicable. }
$$

\section{Competing interests}

Dandan Song, Sohaib Shujaat, Yan Huang, Jeroen Van Dessel, Constantinus Politis, Ivo Lambrichts, and Reinhilde Jacobs declare that they have no competing interests.

\section{Author details}

1OMFS IMPATH Research Group, Department of Imaging \& Pathology, Faculty of Medicine, KU Leuven, Kapucijnenvoer 33, 3000 Leuven, Belgium. ${ }^{2}$ Oral and Maxillofacial Surgery, University Hospitals Leuven, Leuven, Belgium. ${ }^{3}$ West China College of Stomatology, State Key Laboratory of Oral Disease \& National Clinical Research Center for Oral Disease, Sichuan University, Chengdu, China. ${ }^{4}$ Department of Morphology, Biomedical Research Institute, Hasselt University, Diepenbeek, Belgium. ${ }^{5}$ Department of Dental Medicine, Karolinska Institute, Stockholm, Sweden.

\section{Received: 8 August 2020 Accepted: 25 January 2021}

\section{Published online: 18 February 2021}

\section{References}

1. Marco F, Milena F, Gianluca G, Vittoria O. Peri-implant osteogenesis in health and osteoporosis. Micron (Oxford, England : 1993). 2005;36:630-44.

2. Sennerby L, Wennerberg A, Pasop F. A new microtomographic technique for non-invasive evaluation of the bone structure around implants. Clin Oral Implants Res. 2001;12:91-4.

3. Roffi A, Filardo G, Kon E, Marcacci M. Does PRP enhance bone integration with grafts, graft substitutes, or implants? A systematic review. BMC Musculoskelet Disord. 2013;14:330.

4. Albrektsson T, Sennerby L. Direct bone anchorage of oral implants: clinical and experimental considerations of the concept of osseointegration. Int J Prosthodont. 1990;3:30-41 PMID:2372366.

5. De Smet E, Jaecques SV, Jansen JJ, Walboomers F, Vander Sloten J, Naert IE. Effect of constant strain rate, composed of varying amplitude and frequency, of early loading on peri-implant bone (re)modelling. J Clin Periodontol. 2007;34:618-24.
6. Al Subaie AE, Eimar H, Abdallah MN, Durand R, Feine J, Tamimi F, et al. AntiVEGFs hinder bone healing and implant osseointegration in rat tibiae. J Clin Periodontol. 2015;42:688-96.

7. Bissinger O, Probst FA, Wolff KD, Jeschke A, Weitz J, Deppe $\mathrm{H}$, et al. Comparative 3D micro-CT and 2D histomorphometry analysis of dental implant osseointegration in the maxilla of minipigs. J Clin Periodontol. 2017. 44:418-27.

8. Gao Y, Luo E, Hu J, Xue J, Zhu S, Li J. Effect of combined local treatment with zoledronic acid and basic fibroblast growth factor on implant fixation in ovariectomized rats. Bone. 2009;44:225-32.

9. Feldkamp LA, Goldstein SA, Parfitt AM, Jesion G, Kleerekoper M. The direct examination of three-dimensional bone architecture in vitro by computed tomography. J Bone Mineral Res. 1989;4:3-11.

10. Stoppie N, van der Waerden JP, Jansen JA, Duyck J, Wevers M, Naert IE. Validation of microfocus computed tomography in the evaluation of bone implant specimens. Clin Implant Dent Relat Res. 2005;7:87-94.

11. Bernhardt R, Kuhlisch E, Schulz MC, Eckelt U, Stadlinger B. Comparison of bone-implant contact and bone-implant volume between 2D-histological sections and 3D-SRuCT slices. Eur Cells Mater. 2012;23:237-47.

12. Akça K, Chang TL, Tekdemir I, Fanuscu MI. Biomechanical aspects of initial intraosseous stability and implant design: a quantitative micromorphometric analysis. Clin Oral Implants Res. 2006;17:465-72.

13. Bilhan H, Geckili O, Mumcu E, Bozdag E, Sünbüloğlu E, Kutay O. Influence of surgical technique, implant shape and diameter on the primary stability in cancellous bone. J Oral Rehabil. 2010;37:900-7.

14. Germanier Y, Tosatti S, Broggini N, Textor M, Buser D. Enhanced bone apposition around biofunctionalized sandblasted and acid-etched titanium implant surfaces. A histomorphometric study in miniature pigs. Clin Oral Implants Res. 2006;17:251-7.

15. Ruffoni D, Wirth AJ, Steiner JA, Parkinson $I H$, Müller R, van Lenthe $G H$. The different contributions of cortical and trabecular bone to implant anchorage in a human vertebra. Bone. 2012;50:733-8.

16. Albrektsson T. Hard tissue implant interface. Aust Dent J. 2008;53(Suppl 1): S34-8.

17. Romanos GE, Toh CG, Siar CH, Wicht H, Yacoob H, Nentwig GH. Boneimplant interface around titanium implants under different loading conditions: a histomorphometrical analysis in the Macaca fascicularis monkey. J Periodontol. 2003;74:1483-90.

18. De Pauw GA, Dermaut LR, Johansson CB, Martens G. A histomorphometric analysis of heavily loaded and non-loaded implants. Int J Oral Maxillofac Implants. 2002;17:405-12 PMID:12074457.

19. Heitz-Mayfield LJ, Schmid B, Weigel C, Gerber S, Bosshardt DD, Jönsson J, et al. Does excessive occlusal load affect osseointegration? An experimental study in the dog. Clin Oral Implants Res. 2004;15:259-68.

20. Kanno T, Takahashi T, Tsujisawa T, Ariyoshi W, Nishihara T. Platelet-rich plasma enhances human osteoblast-like cell proliferation and differentiation. J Oral Maxillofac Surg. 2005:63:362-9.

21. Jiang N, Du P, Qu W, Li L, Liu Z, Zhu S. The synergistic effect of TiO2 nanoporous modification and platelet-rich plasma treatment on titaniumimplant stability in ovariectomized rats. Int J Nanomedicine. 2016;11:4719-33.

22. Huang Y, Li Z, Van Dessel J, Salmon B, Huang B, Lambrichts I, et al. Effect of platelet-rich plasma on peri-implant trabecular bone volume and architecture: a preclinical micro-CT study in beagle dogs. Clin Oral Implants Res. 2019;30:1190-9.

23. Kilkenny C, Browne WJ, Cuthill IC, Emerson M, Altman DG. Improving bioscience research reporting: the ARRIVE guidelines for reporting animal research. PLoS Biol. 2010;8:e1000412.

24. Huang Y, Dessel JV, Depypere M, EzEldeen M, lliescu AA, Santos ED, et al. Validating cone-beam computed tomography for peri-implant bone morphometric analysis. Bone Res. 2014;2:14010.

25. Huang $Y$, Van Dessel J, Liang X, Depypere M, Zhong W, Ma G, et al. Effects of immediate and delayed loading on peri-implant trabecular structures: a cone beam CT evaluation. Clin Implant Dent Relat Res. 2014;16:873-83.

26. Pearce Al, Richards RG, Milz S, Schneider E, Pearce SG. Animal models for implant biomaterial research in bone: a review. Eur Cells Mater. 2007;13:1-10.

27. Faul F, Erdfelder E, Lang A-G, Buchner A. G* Power 3: A flexible statistical power analysis program for the social, behavioral, and biomedical sciences. Behav Res Methods. 2007:39:175-91.

28. Shin HS, Woo HM, Kang BJ. Optimisation of a double-centrifugation method for preparation of canine platelet-rich plasma. BMC Veterinary Res. 2017;13:198. 
29. de Faria VK, dos Santos CL, da Silveira BM, Laperre K, Padovan LE, Jacobs R, et al. Micro CT assessment of bone microarchitecture in implant sites reconstructed with autogenous and xenogenous grafts: a pilot study. Clin Oral Implants Res. 2017:28:308-13.

30. Soares MQS, Van Dessel J, Jacobs R, da Silva Santos PS, Cestari TM, Garlet $\mathrm{GP}$, et al. Zoledronic acid induces site-specific structural changes and decreases vascular area in the alveolar bone. J Oral Maxillofac Surg. 2018;76: 1893-901.

31. Park YS, Yi KY, Lee IS, Jung YC. Correlation between microtomography and histomorphometry for assessment of implant osseointegration. Clin Oral Implants Res. 2005;16:156-60.

32. Zechner W, Tangl S, Tepper G, Fürst G, Bernhart T, Haas R, et al. Influence of platelet-rich plasma on osseous healing of dental implants: a histologic and histomorphometric study in minipigs. Int J Oral Maxillofac Implants. 2003;18: 15-22 PMID:12608664.

33. Yamazaki T, Sabit H, Oya T, Ishii Y, Hamashima T, Tokunaga A, et al. Activation of MAP kinases, Akt and PDGF receptors in injured peripheral nerves. J Peripheral Nervous Syst. 2009;14:165-76.

34. Davis VL, Abukabda AB, Radio NM, Witt-Enderby PA, Clafshenkel WP, Cairone JV, et al. Platelet-rich preparations to improve healing. Part II: platelet activation and enrichment, leukocyte inclusion, and other selection criteria. J Oral Implantol. 2014;40:511-21.

35. Plachokova AS, van den Dolder J, van den Beucken JJ, Jansen JA. Bone regenerative properties of rat, goat and human platelet-rich plasma. Int J Oral Maxillofac Surg. 2009;38:861-9.

36. Cerciello S, Beitzel K, Howlett N, Russell RP, Apostolakos J, McCarthy MB, et al. The use of platelet-rich plasma preparations in the treatment of musculoskeletal injuries in orthopaedic sports medicine. J Operative Techniques in Orthopaedics. 2013;23:69-74.

37. Alsousou J, Thompson M, Hulley P, Noble A, Willett K. The biology of platelet-rich plasma and its application in trauma and orthopaedic surgery: a review of the literature. J Bone Joint Surg Br. 2009;91:987-96.

38. Davis VL, Abukabda AB, Radio NM, Witt-Enderby PA, Clafshenkel WP, Cairone $\mathrm{JV}$, et al. Platelet-rich preparations to improve healing. Part I: workable options for every size practice. J Oral Implantol. 2014;40:500-10.

39. Peng W, Kim IK, Cho HY, Seo JH, Lee DH, Jang JM, et al. The healing effect of platelet-rich plasma on xenograft in peri-implant bone defects in rabbits. Maxillofac Plast Reconstr Surg. 2016;38:16.

40. Bae JH, Kim YK, Myung SK. Effects of platelet-rich plasma on sinus bone graft: meta-analysis. J Periodontol. 2011;82:660-7.

41. Ilgenli T, Dündar N, Kal Bl. Demineralized freeze-dried bone allograft and platelet-rich plasma vs platelet-rich plasma alone in infrabony defects: a clinical and radiographic evaluation. Clin Oral Investig. 2007;11:51-9.

42. Albanese A, Licata ME, Polizzi B, Campisi G. Platelet-rich plasma (PRP) in dental and oral surgery: from the wound healing to bone regeneration. J Immun Ageing. 2013;10:23.

43. Marx RE, Carlson ER, Eichstaedt RM, Schimmele SR, Strauss JE, Georgeff KR. Platelet-rich plasma: growth factor enhancement for bone grafts. Oral Surg Oral Med Oral Pathol Oral Radiol Endod. 1998;85:638-46.

44. Wiltfang J, Kloss FR, Kessler P, Nkenke E, Schultze-Mosgau S, Zimmermann R, et al. Effects of platelet-rich plasma on bone healing in combination with autogenous bone and bone substitutes in critical-size defects. An animal experiment. Clin Oral Implants Res. 2004;15:187-93.

45. Choi BH, Im CJ, Huh JY, Suh JJ, Lee SH. Effect of platelet-rich plasma on bone regeneration in autogenous bone graft. Int J Oral Maxillofac Surg. 2004;33:56-9.

46. Raghoebar GM, Schortinghuis J, Liem RS, Ruben JL, van der Wal JE, Vissink A. Does platelet-rich plasma promote remodeling of autologous bone grafts used for augmentation of the maxillary sinus floor? Clin Oral Implants Res. 2005;16:349-56

47. Hatakeyama I, Marukawa E, Takahashi Y, Omura K. Effects of platelet-poor plasma, platelet-rich plasma, and platelet-rich fibrin on healing of extraction sockets with buccal dehiscence in dogs. Tissue Eng Part A. 2014;20:874-82. https://doi.org/10.1089/ten.TEA.2013.0058.

48. Marx RE. Platelet-rich plasma: evidence to support its use. J Oral Maxillofac Surg. 2004;62:489-96.

49. Maghsoudi O, Shirazi SH, Abarkar M, Anvar SA. Standardization and moodification techniques of phatelet-rich plasma (PRP) preparation in rabbit. Int Clin Pathol J. 2015;1:5.

\section{Publisher's Note}

Springer Nature remains neutral with regard to jurisdictional claims in published maps and institutional affiliations.

\section{Submit your manuscript to a SpringerOpen ${ }^{\circ}$ journal and benefit from:}

- Convenient online submission

- Rigorous peer review

- Open access: articles freely available online

- High visibility within the field

- Retaining the copyright to your article

Submit your next manuscript at $\boldsymbol{\nabla}$ springeropen.com 